\title{
Causal Effects of Well-Being on Health: It's Complicated
}

\author{
Julia M. Rohrer ${ }^{1} \&$ Richard E. Lucas²
}

In positive psychology and well-being research, subjective well-being is frequently considered a cause of desirable outcomes such as health. We discuss major conceptual complications that cast doubt on existing empirical evidence for such claims. Well-being and health share a multitude of common causes. Neither cross-sectional, longitudinal, nor experimental studies can identify the effects of interest without strong assumptions that are rarely spelled out and that may rarely be plausible. The field should rise to the causal inference challenge posed by the main effects of well-being before moving on to more sophisticated claims concerning underlying mechanisms and boundary conditions.

Philosophers and lay people agree that happiness is highly desirable (Diener, 2000), and it only takes one trip to the local bookstore to realize how appealing the prospect of happiness is to many. This alone is a good reason to consider subjective well-being (SWB) a worthy subject of research. However, many scientists in the field of positive psychology and well-being research go further and claim that SWB is not just a (desirable) epiphenomenon, but also a cause of desirable outcomes. In that spirit, De Neve, Diener, Tay, and Xuereb (2013) provided a list of "objective benefits of subjective wellbeing", including, among others, increased productivity, higher income, reduced consumption, and increased pro-social behavior. The inclusion of each outcome on this list was based on an impressive body of literature.

One topic that has attracted particular interest is the question of whether (and why and when) subjective well-being causally affects health. In their review of the literature, Diener, Pressman, Hunter and Delgadillo-Chase (2017) concluded that SWB can indeed influence health. They pointed out that while all designs have weaknesses, the sum of the evidence is promising and the field is ready to leave the initial stage in which this causal

\footnotetext{
${ }^{1}$ Department of Psychology, Leipzig University. julia.rohrer@uni-leipzig.de

2 Department of Psychology, Michigan State University
} 
effect is simply documented and turn to more sophisticated questions, such as which factors moderate and mediate these effects.

We commend Diener et al. (2017) for their lucid discussion of the weaknesses and strengths of various designs. However, we disagree when it comes to one central claim: Diener et al. (2017) argue that different research designs complement each other; thus, convergence across methodologies suggests that there is good evidence for causal effects of SWB on health. Triangulation, the strategic use of different approaches to address one question, is indeed central for science. However, for it to result in strong evidence, different approaches need to suffer from unrelated sources of bias (Krieger \& Davey Smith, 2016; Munafò \& Davey Smith, 2018). Here, we will argue that common designs employed in the literature on the effects of SWB on health suffer from the same bias, that is, highly plausible confounding by a multitude of common causes. Amassing a large number of studies that share the same bias does not do much to rule out the possibility that findings are spurious. It may even be harmful insofar it can create the illusion of a robust evidence base when studies are predictably reproducing the same confounded pattern.

We will start with a clarification of what claims about causal effects of SWB entail, and subsequently discuss major obstacles to the identification of such effects in cross-sectional, longitudinal, and experimental designs. All of the points we discuss apply equally to studies that claim that SWB does have an effect on health and to studies that claim that SWB does not have effect on health; our focus is on the former type of study as it seems to be more prevalent in the published literature, and as the biases we discuss will tend to induce spurious positive associations. We conclude with some potentially productive ways forward for this field of inquiry, stressing the value of spelling out assumptions and highlighting the need for more conceptual work.

In the following, we will use the term subjective well-being to refer to a broad construct that captures how individuals evaluate their own lives.

\section{What does a Causal Effect of SWB Mean?}

It is easy to get lost in debates about the definition of causality, and the case can be made for pluralism in causal inference (e.g., Krieger \& Smith, 2016). That said, good starting points include coherent frameworks that allow researchers to be more precise about causal claims (see Morgan \& Winship, 2015 for an excellent and broad introduction). For 
psychologists, the potential outcomes model may be particularly intuitive because it was originally developed in an experimental context. In the potential outcomes model, the individual-level causal effect of a treatment is the difference between the individual's outcome, had they experienced the treatment, and the individual's outcome, had they experienced the control state. Considering the effects of SWB on health, you may for example wonder what your overall health will look like in one year if you were very happy today (or very happy on average across some longer time span), compared to if you were only moderately happy. The fundamental problem of causal inference is that we can only observe one of these outcomes and hence cannot directly estimate the causal effect for any particular individual. However, under certain assumptions which we will discuss below, other quantities can be estimated, such as the average treatment effect across individuals.

This helps us to clarify what would not be deemed a causal effect of SWB. We will now switch to a graphical framework, which makes it easier to reason about different causal situations (for an introduction to this approach, see Rohrer, 2018) but is equivalent to the potential outcomes model (Elwert, 2013). Figure 1 is a very simple directed acyclic graph visualizing SWB, three of its causes (e.g., personality, socio-economic status, social support) and health. The thin arrows alone-factors that causally affect both SWB and health-do not imply a causal effect of SWB on health; instead they reflect confounding factors. These associations merely indicate that what makes us happy also makes us healthy. The bold arrow pointing directly from SWB to health represents what we mean when we talk about a causal effect of SWB on health: being happier makes us healthier, regardless of the factors that made us happy. In particular, assuming that there is a causal effect of SWB on health, if we had an extremely precise intervention that would only manipulate SWB (but none of its causes), this intervention would have effects on subsequent health. ${ }^{3}$

\footnotetext{
${ }^{3}$ Here, we subscribe to the notion that we can define a causal effect of SWB regardless of the source of change in SWB, which is aligned with a structural causal model framework (e.g., Pearl, 2018). Not every causal inference expert agrees with this viewpoint (e.g., Hernán \& Taubman, 2008). One concern is that the assumption of treatment-variation irrelevance (Hernán \& Robins, 2010, p. 36) may be violated. For example, a 1-point increase in SWB may have different effects depending on how it was achieved (e.g., through drugs or through social interactions). In such a scenario, it would not make sense to talk about the effect of SWB on health.
} 


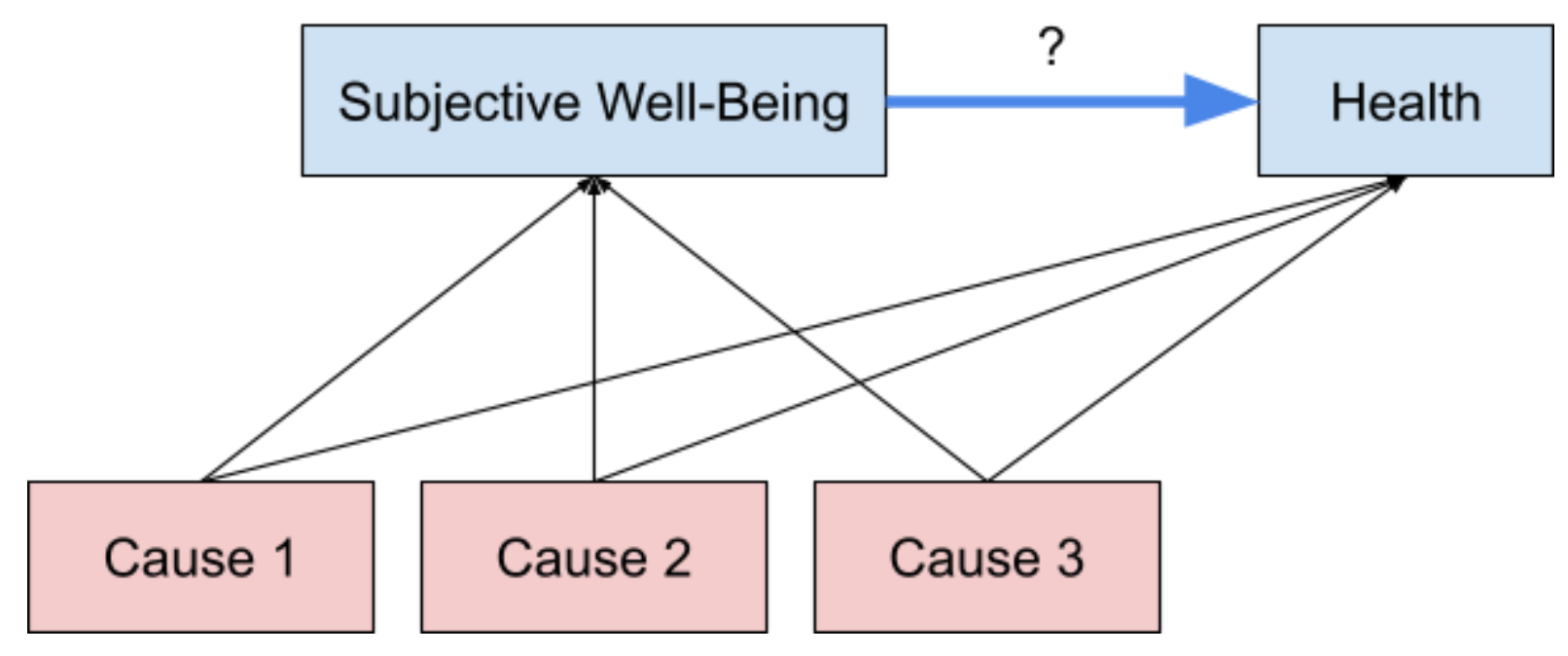

Figure 1. The fundamental problem: common causes confound the correlation between well-being and health.

To establish the bold arrow, we need to rule out two important alternative explanations: first, that the arrow actually points from health to SWB, i.e., that the association of interest is attributable to the fact that health causes SWB; second, that the association of interest is attributable to common cause confounding.

\section{Different Research and Analysis Designs}

\section{Cross-sectional correlations}

Observing only the cross-sectional (zero-order) correlation between SWB and health does not allow us to rule out either alternative explanation. Temporal order may help us rule out the first alternative explanation. But to rule out the second alternative explanation, control variables are necessary. If we were able to control for all shared causes of SWB and health (e.g., personality, socio-economic status, social support and other life circumstances, but also prior health), we could successfully identify the causal effects of SWB on health. But the potential joint causes of SWB and health are numerous ${ }^{4}$ and thus, researchers might not be willing to assume that they have thought of and measured all of them. Instead, they may choose more sophisticated approaches, such as longitudinal data and experimentation.

\footnotetext{
${ }^{4}$ Indeed, one of the strengths of the SWB concept is that it subsumes so many different causes-but this strength turns out to be a nuisance when we want to conceptualize SWB as the independent variable.
} 
However, as we will discuss in the next sections, these designs nonetheless rely on the assumption of no unobserved confounding.

\section{Longitudinal Data}

Longitudinal models are often considered a viable solution to problems of causal inference. Their advantage is twofold: First, they remedy concerns about reverse causality; second, they allow for within-subject analyses that rule out between-subject confounders. For example, if a study finds that for a particular individual, a change in well-being precedes a change in health, this cannot be attributed to reverse causality. And it also cannot be attributed to stable factors (e.g., stable measures of personality, aspects of socio-economic status that have not changed over the course of the study, childhood experiences predating the study). In psychology, cross-lagged panel models are the most popular choice for longitudinal data, although it should be noted that some modifications are necessary to ensure that between-subject confounders are indeed ruled out (such as the inclusion of random intercepts, Hamaker et al., 2015)

However, even with modifications, cross-lagged panel models only aim at so-called "Granger causality" (as acknowledged in footnote 1 of Hamaker, Kuiper, \& Grasman, 2015). Granger causality occurs when a time series of one variable X (e.g., SWB) is able to predict future values of another variable $Y$ (e.g., health) beyond current values of $Y$ (Granger, 1969). But Granger causality is not the same as causality, ${ }^{5}$ and it is easy to come up with situations in which Granger causality does not imply that $X$ causally affects $Y$. Consider, for example, a third variable like respondent's financial situations. Improvements in the financial situation might cause almost immediate increases in SWB via improved mood, but the effects on health may take longer to unfold-for example, additional income may allow respondents to improve their diets and reduce stress levels over the course of a few years. In such a scenario, SWB "Granger-causes" health, as SWB contains information about the financial situation (which affects future health), but SWB may have no causal effect on health. Thus, longitudinal data does not automatically rule out all confounding factors-it only rules out between-subjects confounding. We still need to consider the possibility of within-subject

\footnotetext{
${ }^{5}$ Supposedly, the late Clive Granger admitted that he felt embarrassed by the term "Granger causality" as it had nothing to do with causality, but he could not stop people from using it (Pearl, 2019).
} 
confounding, which may often be highly plausible for psychological constructs that change over time.

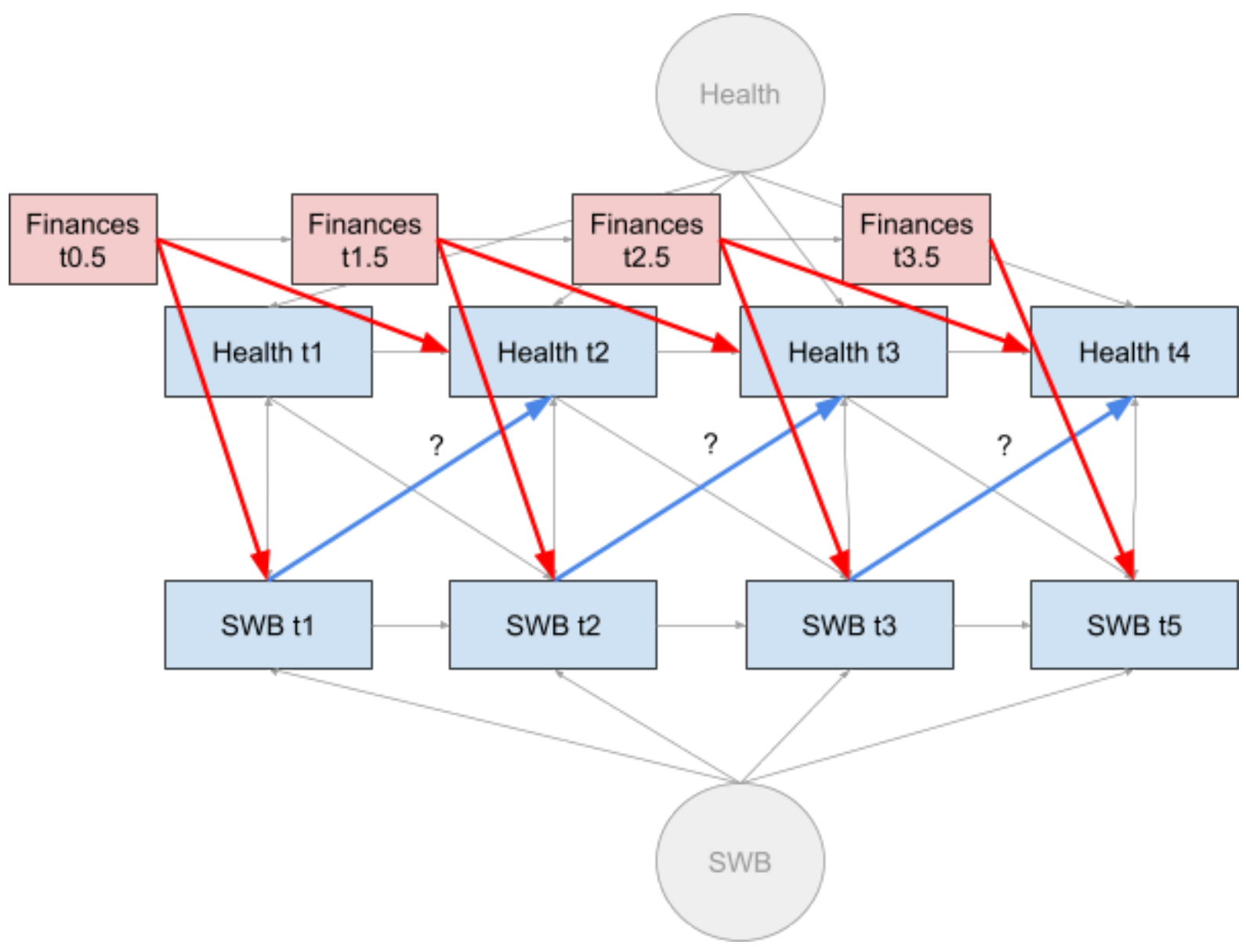

Figure 2. Longitudinal confounding in a random-intercept cross-lagged panel model. The estimate of the effect of interest (SWB $\rightarrow$ Health) is biased unless we take into account the time-varying confounder (Finances).

As a side note, cross-lagged models may seem particularly appealing because they capture the notion of feedback loops: SWB affects health, which in turn affects SWB, which in turn affects health, and so on. This notion is in line with the view that humans are complex dynamical systems-a perspective that may be conceptually fruitful, but that unfortunately does not simplify matters of causal inference. Common network models, the statistical approach most strongly associated with a dynamic systems view in psychology, only provide a statistical representation of the underlying variables, not a causal one (Dablander \& Hinne, 2019); moving to causality once again requires strong assumptions. Even just the generation of causal hypotheses with the help of such causally-agnostic network models poses 
challenges (Ryan et al., 2019). Of course, network models are not synonymous with dynamic systems, and one may consider different, more inclusive conceptions of causality in such systems (e.g., "coupling", van Geert, 2020). While this could be a productive path in the context of research on subjective well-being, it would require challenging conceptual groundwork to ensure that "complexity" is not used in a vague sense to deflect criticism and to create a smokescreen (see e.g. Friedman \& Brown, 2018 for a cautionary tale).

\section{Experimental Interventions}

Correlation does not imply causation, and so psychologists run experiments. In an experiment, the treatment is randomly assigned; hence, it cannot be confounded with the outcome. However, in the case of SWB, the complication arises that we cannot randomly assign individuals to different levels of well-being - it is a non-manipulable cause. We can, at best, randomly assign them to receive some intervention which in turn (hopefully) affects their SWB. We highlight an exemplary study that took this approach in Box 1.

\section{Box 1: A Randomized Trial of Happiness}

Kushlev et al. (2020) conducted a trial in which participants were randomly assigned to participate in a comprehensive 3-month positive psychology intervention (vs. a wait-list control group). The study exemplifies many best practices (randomized treatment; high transparency including pre-registration, open data, and reporting of null results), and we are discussing it here to illustrate how complex causal inference issues affect even the most well-conducted studies. The authors found that their treatment, which did not target any health behaviors, had an effect on self-reported health (but not on two objective measures, BMI and blood pressure), and this effect is causally identified thanks to randomization.

But are the changes in health caused by changes in SWB? To further address this question, the authors predicted sick days in a given week from the difference in SWB between that week and the previous week, controlling for sick days during the previous week. This analysis does not directly map onto the types of analyses that we discuss in the main text, but a conceptual graph can clarify the matter (Figure 3). Note that the change in SWB is an outcome of SWB in the present week-even though one may conceptualize it as occuring in between weeks, it is a deterministic function of the two 
SWB scores. The intervention is not represented in the graph; it does, in fact, not explicitly show up in the respective analyses.

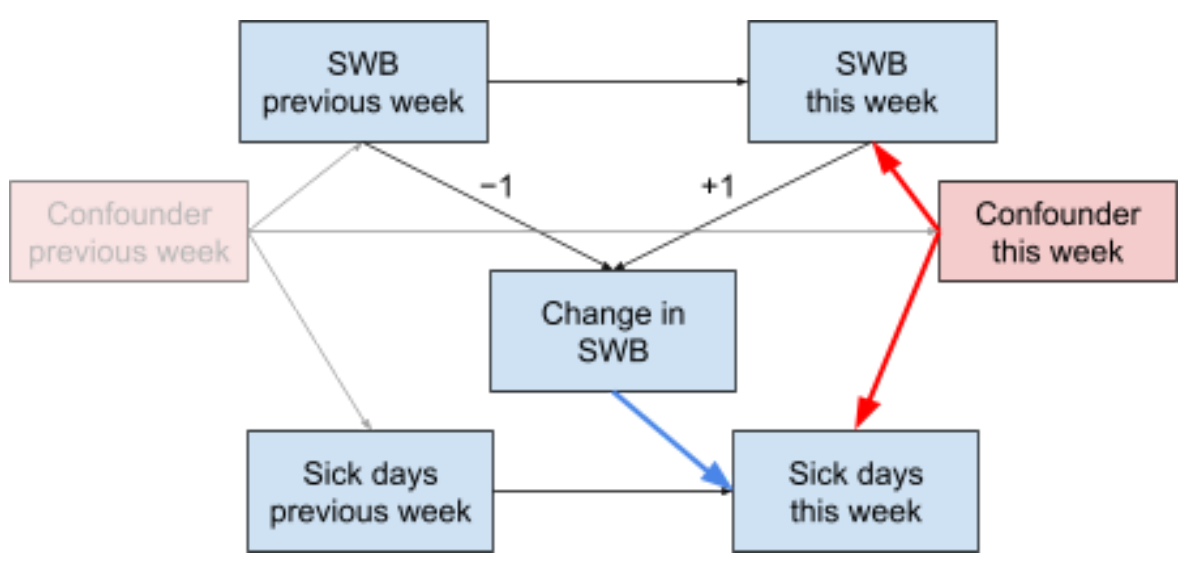

Figure 3. Conceptual graph of the analyses reported by Kushlev et al. (2020) in which sick days are predicted from the change in well-being from last week to this week.

Can we identify the causal effect of a change in SWB on sick days in the current week? If there are any confounding variables (e.g., workplace stress) that affect both SWB and sick days, there will be a confounding path: Change in SWB $\leftarrow$ SWB this week $\leftarrow$ Confounder this week $\rightarrow$ Sick days this week. This path will introduce a spurious association between Change in SWB and Sick days this week, unless we control for the confounder. There is also a second confounding path: Change in SWB $\leftarrow$ SWB previous week $\leftarrow$ Confounder previous week $\rightarrow$ Confounder this week $\rightarrow$ Sick days this week. Thus, the reported analyses can (once again) only successfully identify the causal effect of interest if all relevant confounding variables have been controlled for.

Can such studies fix our causal inference problem? Unfortunately, the intervention is almost guaranteed to affect other variables as well. SWB interventions are typically designed to affect SWB through various variables (e.g., social relationships, physical activity, mindfulness) and each of these might plausibly explain any effect of the treatment on the outcome of interest (e.g., health).

In an attempt to isolate the effects of SWB from the effects of other variables affected by the treatment, researchers may run a mediation analysis to identify the effect of the treatment mediated via SWB. However, mediation analysis makes its own strong assumptions (see Imai, Keele, \& Tingley, 2010 for an introduction to causal mediation 
analysis)—among them the assumption that all common causes of the mediator and the outcome are accounted for. In our scenario, this means that we need to assume that we have controlled for all common causes of SWB and health. But this is exactly the strong assumption that we wanted to avoid, and any violation may lead to spurious indirect effects.

For example, let us assume that an experimental intervention had a causal effect on both SWB and health. To estimate the indirect effect of the intervention on health via SWB, we need to multiply the effect of the intervention on SWB with the effect of SWB on health. If SWB and health were confounded (Figure 4), for example by participants' socio-economic status, the estimate of the latter would be biased, and as a result, the estimate of the indirect would be biased (unless we controlled for the confounder).

So mediation analysis does not fix our causal inference problem-in fact, we need to fix our causal inference problem before we can run an interpretable mediation analysis. In general, psychologists seem to underestimate the challenges that mediation analysis raises even in experimental contexts (Bullock et al., 2010).

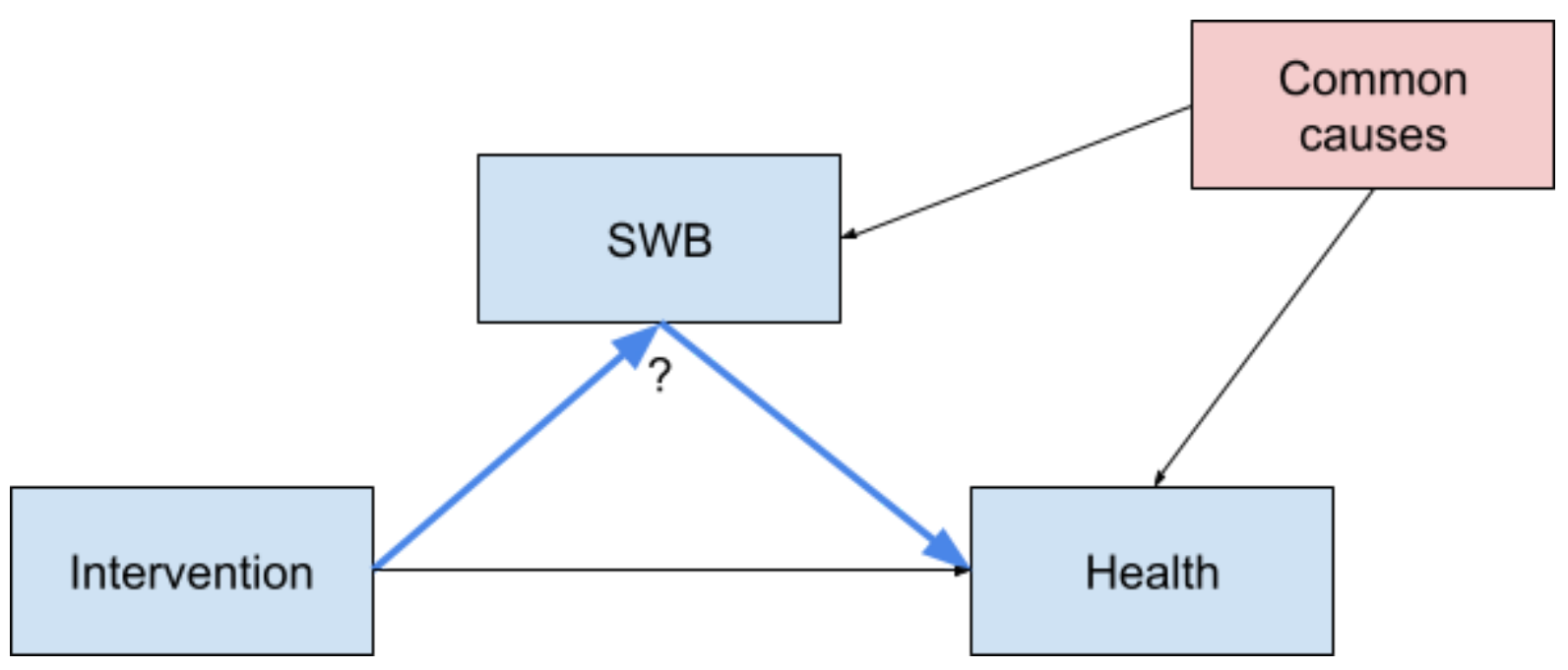

Figure 4. In mediation analysis, confounding can induce spurious indirect effects, even if the treatment has been randomized.

Where psychologists default to mediation analysis, economists may instead apply instrumental variable estimation (see Grosz et al., 2020 for a brief introduction for psychologists). In contrast to standard mediation analysis in psychology, instrumental variable estimation is quite explicitly framed as a tool for causal inference. In our case, we could use our intervention as a so-called "instrument" to induce random variation in SWB, which in turn can be used to identify its effects on other variables (e.g., health). If this approach is successful, it returns the average causal effect of SWB on health among 
"compliers" (i.e., the people for which participation in the intervention has a positive effect on SWB). But for it to succeed, three assumptions must be met.

The exchangeability assumption demands that the instrument does not share a common cause with the outcome. This can be guaranteed if the instrument is a randomized intervention which cannot possibly be confounded with anything.

The relevance assumption demands that the instrument must have a causal effect on the variable whose effects should be identified. In the case of the effects of SWB on health, we thus need an intervention that causally affects SWB-ideally in a strong and reliable manner, which is a challenge in its own right (e.g., White et al., 2019).

The exclusion restriction demands that the instrument affects the outcome only through the variable whose effect should be identified (Figure 5). This is the crucial issue for the current application: The intervention must affect health only through SWB. If, for example, the intervention has beneficial effects that are mediated through other channels than SWB (e.g., social relationships, physical fitness, mindfulness), it no longer is a valid instrument. Additional paths may be blocked through statistical control, but this once again leaves us in a situation in which third variables need to be carefully considered. 


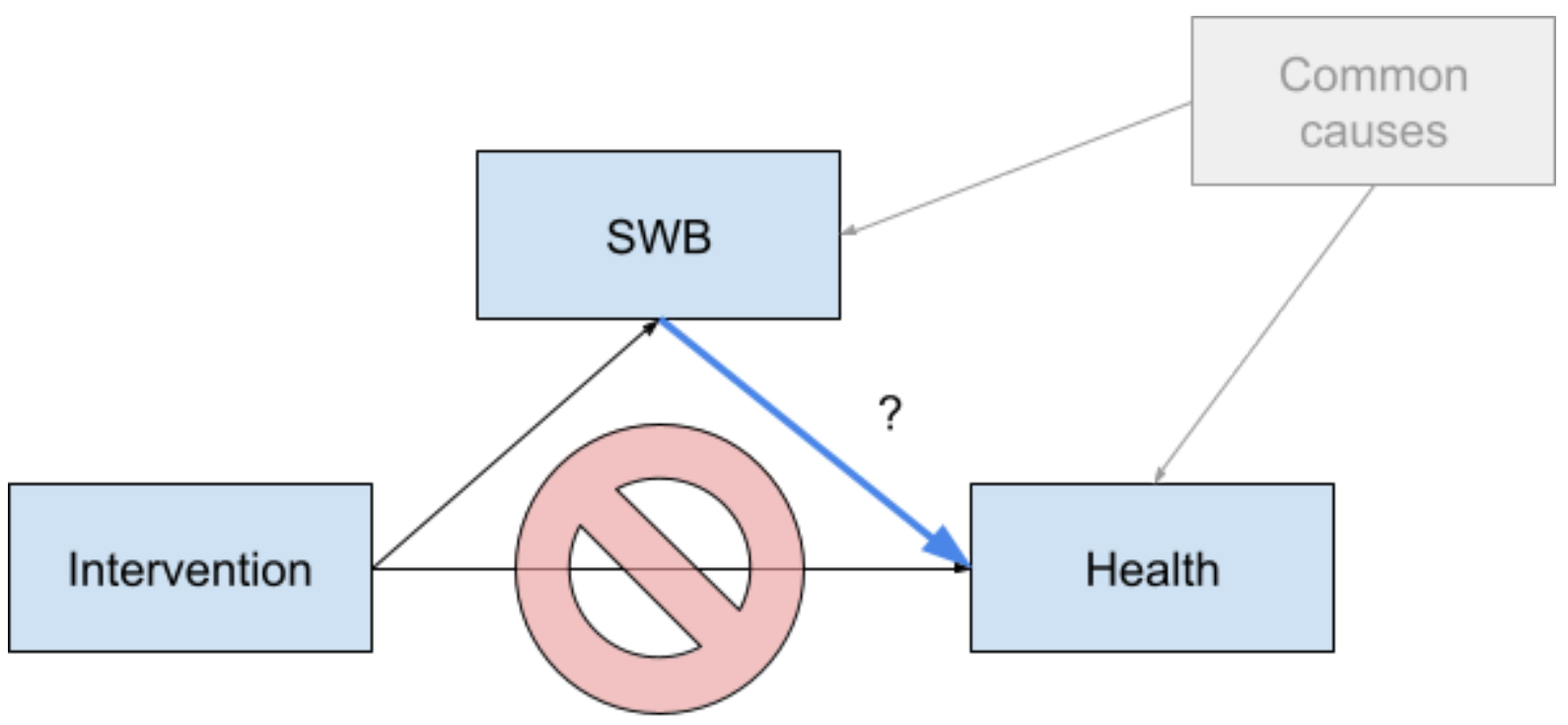

\section{Exclusion restriction: the intervention only affects health through its effect on SWB}

Figure 5. Using an intervention as an instrument to identify the causal effects of SWB. Here, unobserved common causes of SWB and health are no longer a concern, but the crucial assumption is that the intervention only affects health through its effect on SWB (i.e., full mediation).

The problem, then, is that we want something that is (1) effective enough to reliably change SWB but (2) not so broad as to affect many other variables which may in turn affect health. Consideration of both mediation analysis and instrumental variable estimation leads to the same conclusion: We need a highly targeted intervention, akin to strong mood enhancers, without further (physiological or psychological) side effects. Such an intervention may not be realistically possible, but see Box 2 for one potentially promising class of instruments: genetic variants.

Reflecting on the exclusion restriction may help us discover effects that can be causally identified more easily. For example, if we replace both SWB and health with narrower constructs, it may become easier to find a valid instrument. If one was interested in identifying the effects of positive mood on subsequent short-term cardiovascular function, an experimental manipulation (e.g., showing an uplifting movie scene) may be a plausible instrument fulfilling the necessary assumptions. But if we wanted to move from such 
narrower findings to broader conclusions, we would once again face considerable inferential issues (e.g., Yarkoni, 2019).

Importantly, these concerns about mediation analysis and instrumental variable estimation only apply if the goal is to identify the effects of SWB on health. If instead the goal is to establish that a certain intervention affects health (or any other outcome) regardless of the mechanism, these specific complications do not matter.

\section{Box 2: A Mendelian Randomization Study on SWB and Health}

A promising approach to causal inference that is rarely encountered in the field of well-being research leverages genetic information to rule out common causes (see Briley et al., 2018 for an introduction). For example, Wootton et al. (2018) conducted a so-called mendelian randomization study to investigate whether SWB affects cardiometabolic health (and vice versa). Mendelian randomization takes the logic of instrumental variables to the genetic level. If we know that a genetic variant has an effect on SWB, it can potentially serve as an instrument to identify the causal effect of SWB on health. The same assumptions as for other instrumental variables apply. For example, the exclusion restriction may be violated if the genetic variant affects health through other pathways as well, although such concerns can be partly addressed through the usage of a large number of genetic variants and through multiple estimation methods. In their study, Wootton et al. found no evidence that SWB affects any of the investigated cardiometabolic health measures. However, their instrument was relative weak-genetic variants only accounted for about $0.01 \%$ of the variance in subjective well-being-and so the finding may be false negative. The authors did find evidence for a causal effect that runs into the other direction: body mass index had a negative effect on well-being, more specifically, satisfaction with health.

\section{How much Control is Enough?}

So far, we have been rather vague when referring to potential confounders, and one may criticize us for this lack of specificity. If, instead, we provided a list of the specific confounders that are most important, researchers could move forward by probing whether controlling for them removes the effects of SWB on health. In fact, for almost any potential confounder, there may already be a study out there demonstrating that it cannot explain 
away the effects of SWB on health. Would that be sufficient to demonstrate that SWB does indeed causally affect health?

A recent review of the literature (Diener et al., 2018) provides a comprehensive list of factors that are assumed to affect SWB. The factors that typically emerge as the strongest predictors of well-being-including variables like socioeconomic status, social relationships, and specific personality traits-are often found to be robust predictors of health. Thus, they may be considered potential confounders. For confounding to be a substantial problem, it is not necessary to single out central variables. Any single confounder may have only very small effects on both SWB and health; but if there are enough of them, the resulting bias will be relevant. This argument assumes that the biases mostly point into the same direction, which seems plausible for the case of SWB and health: "Good things" make us both happy and healthy. In such a scenario, any study demonstrating that a confounder (or any limited set of confounders) does not fully explain away the effects of SWB on health does not do much to relief our concerns regarding confounding. We would need a study that simultaneously considers all relevant confounders. To make matters worse, even if all relevant confounders had been included, measurement error may still result in residual confounding (Westfall \& Yarkoni, 2016).

This may make it sound as if sufficient control was an impossible requirement. Indeed, in a correlational study, the claim that unobserved confounders are a problem constitutes an irrefutable criticism, and so we can never be certain about conclusions. But certainty is not necessary for knowledge: rigorous studies accounting for many alternative explanations do increase the plausibility of a causal effect. In Box 3, we provide a brief review of longitudinal studies investigating the effects of SWB on health with a focus on how well they account for alternative explanations. In brief, we believe that given the current state of the literature, there is a lot of room for incremental improvement.

\section{Box 3: A Brief Review of Longitudinal Studies}

Diener et al. (2017) provided a list of 19 longitudinal studies, which we reviewed with an eye on four criteria.

Are the data analyzed in a manner that is meant to address causal questions? Not all of the studies seem to aim at (Granger) causality; for example, three of them investigate 
whether changes in SWB are correlated with changes in health (Kim et al., 2014; Ryff et al., 2015; Seale et al., 2010) and one applied a fixed-effects analysis (Fischer \& Sousa-Poza, 2009). Under the plausible assumption that health affects well-being, these analyses cannot identify the causal effect of well-being on health.

Is the measure of SWB not directly confounded with health? For example, Voellmin et al. (2013) investigated the effects of maternal positive affect on pregnancy outcomes (e.g., preterm delivery). However, the measure of maternal positive affect included 7 positive and 6 negative feelings towards pregnancy, assessed during pregnancy. Here, the parsimonious alternative explanation would be that pregnancy complications affect both maternal feelings towards pregnancy and pregnancy outcomes. Similarily, Ickovics et al. (2006) investigated whether psychological resources affected HIV-related outcomes over a five-year period, but their resources included "Positive HIV Expectancy." Here, the alternative explanation is that individuals' expectancies are at least partially affected by accurate information about their health status. Other studies used measures that blended traditional SWB measures with other constructs, which changes the target of the analysis.

Are basic confounders accounted for? These may include basic demographic characteristics and income, initial health status and health behaviors, and other factors that have been identified as potential causal influences on both SWB and health such as personality and social relationships. Although most (but not all) studies controlled for at least some demographic characteristics (most commonly age, gender, education, and race/ethnicity), few studies controlled for variables from more than two or three categories. Only about one-half of studies controlled for either of the two most obvious confounds—income or prior health—and almost no studies controlled for personality or social relationships.

Is the statistical evidence strong? While statistical reliability is not sufficient to establish causality, it is a necessary precondition for taking empirical evidence seriously. Only about half of the studies reported a focal $p$ value below .01 (as opposed to being between 01 and .05 or nonsignificant). For instance, in their examination of whether positive affect predicts HIV viral load, Carrico and Moskowitz (2014) did not even find significant total over-time associations with the outcome; support for their hypotheses only came from significant indirect effects through HIV care and anti-retroviral therapy persistence. Moskowitz (2003) found that positive affect predicts lower risk of AIDS mortality, but the 
predictor (positive affect) may actually be impacted by declining health when death is imminent. Thus, they additionally ran lagged analyses to rule out that positive affect simply tracks the downturn of physical health. The effect of interest became weak when lagged by 12 months and nonsignificant when lagged by 24 months or more. Other studies, including those by Pressman and Cohen (2012) and Kim, Park, et al. (2014), had effects very close to .05 after the inclusion of relevant controls. This pattern does not imply that questionable research practices affected any single study; however, it raises concerns about the reliability of the evidence across studies. Most of the studies in this list use different predictors and outcomes, so there is little evidence of robust, replicable associations across studies.

Not a single of the 19 study met our four criteria. Even when relaxing demands for plausible causal identification, our brief review suggests that few existing studies provide strong evidence for causal effects of SWB on health. The full review, including a table summarizing the central features of each study, can be found on the OSF (https://osf.io/k7egw/).

\section{How much Control is too much?}

Our emphasis on the multitude of confounders may raise concerns regarding "overcontrol." It has been argued that including certain covariates may hide the benefits of SWB (e.g., Diener, Pressman, \& Lyubomirsky, 2015). Indeed, there are situations in which certain third variables should not be controlled for (Rohrer, 2018). However, in practice, researchers may simply notice that expected associations often disappear or are no longer statistically significant when certain third variables are controlled for and thus conclude that harmful overcontrol occured, or that multicollinearity is an issue (Vanhove, 2020). It may thus be helpful to clarify what overcontrol does (or does not) mean.

The purpose of the statistical control of confounders is to get unbiased estimates of a causal effect. If the inclusion of a third variable that is plausibly a confounder-a cause of both the independent and the dependent variable of interest-removes or diminishes the effect of interest, that is not a reason to claim that overcontrol is an issue. However, if the third variable is a plausible mediator (or a collider) — a variable that is causally affected by the independent variable-overcontrol becomes an issue. The induced bias may make significant associations disappear, but it can also induce or amplify associations. As a rule of 
thumb, variables that are causally affected by the dependent variable of interest should not be controlled for. Overcontrol is thus defined by the causal web underlying the variables. In this spirit, Diener, Pressman and Lyubomirsky (2015) argue that sleeping better, smoking less and exercising more are outcomes of higher well-being and thus should not be controlled for when investigating the effects of SWB on health. Assuming that SWB does affect health behaviors, this is indeed a valid concern of overcontrol.

But these examples reflect a situation that is more complicated, because we are talking about third variables which may reflect both confounding factors and mediators at the same time. For example, consider a study in which researchers have assessed SWB, health and health behaviors at a single point in time. They may plausibly argue that health behaviors are a confounder, as they are likely to affect health and possibly SWB. At the same time, health behaviors could be a mediator-maybe happiness gives individuals the necessary energy to go outside and do sports, which in turn affects their health.

Researchers may be tempted to try both analyses-one time controlling for health behaviors, the other time ignoring the variable or even incorporating it as a mediator-and then simply report whatever conclusion they prefer. Running both analyses may be sensible, but if estimates differ substantially depending on whether or not health behaviors are controlled, one cannot simply choose the analysis that best fits the preferred causal model. Instead, these decisions must be justified. In this case, that would mean providing reasoned arguments and ideally empirical evidence supporting the idea that health behaviors do not reflect confounders. In cases where such arguments cannot be made, one may unfortunately have to conclude that the data available are uninformative and thus do not provide evidence for or against the causal effects of SWB on health.

\section{Productive Ways Forward}

When we challenge claims about the causal effects of SWB on health, we do not mean to diminish the importance of SWB as a topic of study. SWB is an immensely valuable outcome variable; it captures an almost infinite set of causal variables. SWB is a global judgment about one's life as a whole and it allows respondents to consider (explicitly or implicitly) any feature of their life that might contribute to its quality (Diener et al., 2018). This very strength of the concept turns into a weakness when it comes to identifying causal effects of SWB. Each of the factors that contribute to well-being judgments might also serve as a confound that partially explains the link between SWB and outcomes like health. But 
controlling for the almost infinite combination of potential confounds becomes an insurmountable task. While many causal factors are challenging to study, the very nature of SWB -its broad inclusiveness - makes it extremely hard, if not impossible, to design studies that establish its causal effects on other outcomes under reasonable assumptions.

As we have explained above, neither longitudinal data nor experimentation provide direct solutions for this problem. The pervasive nature of potential confounders means that this is not merely a "more research is needed" type of situation-even if there are thousands of studies supposedly illustrating the effects of SWB on health, they provide little evidence if they all suffer from the same bias. This does not mean that we should conclude that SWB has no effects on health. The issue of confounding simply means that the body of existing studies may not be diagnostic, despite its size. How can researchers address this situation in a productive manner?

\section{Making Assumptions Transparent}

First, we suggest that researchers should be more transparent about assumptions underlying their causal claims (including claims about the lack of a causal effect) and they should demand more transparency when acting as reviewers. This approach is different from simply refraining from (explicit) causal claims, which is probably of little use given that many analytic approaches are highly suggestive of causal effects. Euphemisms for causality ("SWB longitudinally predicts...", "changes in SWB are associated with...") do little to prevent others from arriving at causal conclusions (Grosz, Rohrer, \& Thoemmes, 2020). Instead, researchers should feel free to acknowledge that their goal is causal inference, and should explicitly list the concrete underlying assumptions. For example, if researchers rely on a random intercept cross-lagged panel model, they may write that "Results provide evidence for a causal effect of SWB on health under the assumption that there are no time-varying confounding factors that affect both SWB and health with different time lags." This not only allows readers to assess for themselves whether they are willing to accept these assumptions and follow the authors' conclusion, but may also increase awareness of assumptions underlying popular models.

Assumptions may also be supplemented with so-called sensitivity analyses. In the context of causal inference, these analyses quantify to which extent results are sensitive to violations of the underlying assumptions. How strong would confounding need to be to explain away the observed association? Different methods have been developed to answer 
this question in different contexts (e.g., Oster, 2019; Robins et al., 2000), including mediation analysis (Imai et al., 2010; MacKinnon \& Pirlott, 2015).

\section{Clearer Conceptualizations}

Second, there is conceptual work to do. We would like to see researchers who are invested in the idea that there are beneficial effects on SWB on other variables rise to the challenge and to develop defensible causal identification strategies. So far, the intricacies of causal inference have mostly been neglected within the field, leading to what we consider premature certainty in the magnitude of effects. Assuming that convincing causal claims are the designated goal of our studies, researchers can only profit from learning more about the fundamentals of causal inference. This may require drawing on sources outside of psychology, as the approach to causal inference in psychology tends to be unsystematic and often does not benefit from recent developments in other fields of research.

A thorough conceptual consideration of the matter may result in a plausible and defensible identification strategy, or in strategies that at least allow us to rule out a wide range of within-subject confounders. Such a strategy would be a scientific contribution in its own right, regardless of whether it can be implemented immediately, and regardless of whether it leads to the conclusion that SWB does affect health-or not. Alternatively, a conceptual investigation may result in the conclusion that such strategies do not exist, or even that claims about such causal effects are not well-defined to begin with. This may be an ultimately unsatisfying conclusion for many, but at least it would prevent researchers from pursuing lines of research that cannot result in clear answers because they do not pose clear questions.

Calls for conceptual work are necessarily somewhat vague, so let us suggest two concrete starting points.

Approaching the issue from a structural model perspective, researchers may try to summarize our knowledge concerning the causal net underlying subjective well-being and health. Such an endeavor may result in a comprehensive graph capturing the field's combined knowledge and assumptions, which can in turn be used to generate a viable causal identification strategy (see e.g., Rohrer, 2018 for an introduction to this approach)

Approaching the issue from an intervention-oriented perspective, researchers may try to design a so-called target trial (Hernán \& Robins, 2010, pp. 37-40). A target trial describes 
a (hypothetical) experiment, including eligibility criteria and a (hypothetical) treatment strategy. The specification of such a trial results in a more precise definition of a causal effect of interest-even if the trial cannot possibly be implemented, it provides a guideline for an analysis of observational data that aims to emulate the trial. As noted by Hernán and Robins (2010, pp. 34), increasing the precision of the definition of a causal effect may sometimes alter the original research question-this may happen for the case of SWB and health, where both constructs are rather broad and somewhat vague. "The effect of SWB on health" may just be a starting point for a discussion in the field resulting in more specific research questions.

\section{Let's not get Ahead of Ourselves}

Third, we urge researchers to resist the pressure to make increasingly sophisticated claims about the association between SWB and health. While claims about mediators and moderators are more satisfying intellectually, establishing them empirically is even more complicated than establishing simple "main" effects. Causal mediation analysis is very hard, and the predominant approach in psychology ignores recent developments in the field (e.g., Imai, Keele, \& Tingley, 2010). Moderation analysis only makes sense if the effect to be moderated is causally identified, and even then, the causal role of the moderator may be unclear (Rohrer \& Arslan, 2020; VanderWeele, 2009). Increasingly sophisticated claims in combination with a lack of understanding of the underlying assumptions can lead to unfortunate dynamics. On the basis of the published literature, researchers may erroneously conclude that more basic claims are firmly established and thus do not require critical investigation, and they may (probably correctly) conclude that sophisticated claims are necessary to get published, which disincentivizes more rigorous attempts to answer fundamental questions.

We suggest that as a field, we should instead try to walk before we run.

\section{Acknowledgments}

Stefan C. Schmukle and Ed Diener provided helpful feedback on an earlier version of this manuscript. On December 16, 2020, we discussed an earlier version of this manuscript in a digital journal club organized by Peder Isager and Daniel Lakens. The input from participants of this event led to further changes. Kostadin Kushlev and Robyn E. Wootton both provided feedback to ensure that their respectie studies were summarized accurately. 


\section{References}

Briley, D. A., Livengood, J., \& Derringer, J. (2018). Behaviour genetic frameworks of causal reasoning for personality psychology. European Journal of Personality, 32(3), 202-220.

Bullock, J. G., Green, D. P., \& Ha, S. E. (2010). Yes, but what's the mechanism?(don't expect an easy answer). Journal of Personality and Social Psychology, 98(4), 550.

Carrico, A. W., \& Moskowitz, J. T. (2014). Positive affect promotes engagement in care after HIV diagnosis. Health Psychology: Official Journal of the Division of Health Psychology, American Psychological Association, 33(7), 686-689.

Dablander, F., \& Hinne, M. (2019). Node centrality measures are a poor substitute for causal inference. Scientific Reports, 9(1), 6846.

De Neve, J., Diener, E., Tay, L., \& Xuereb, C. (2013). The Objective Benefits of Subjective Well-Being. In World happiness report. https://papers.ssrn.com/abstract=2306651

Diener, E. (2000). Subjective well-being. The science of happiness and a proposal for a national index. The American Psychologist, 55(1), 34-43.

Diener, E., Lucas, R. E., \& Oishi, S. (2018). Advances and Open Questions in the Science of Subjective Well-Being. Collabra. Psychology, 4(1). https://doi.org/10.1525/collabra.115

Diener, E., Pressman, S. D., Hunter, J., \& Delgadillo-Chase, D. (2017). If, Why, and When Subjective Well-Being Influences Health, and Future Needed Research. Applied Psychology. Health and Well-Being, 9(2), 133-167.

Diener, E., Pressman, S. D., \& Sonja, L. (2015, December 17). Op-Ed: Can 1 million women be wrong about happiness and health? Los Angeles Times. https://www.latimes.com/opinion/op-ed/la-oe-lyubomirsky-et-al-happiness-affects-health20151217-story.html

Elwert, F. (2013). Graphical Causal Models. In S. L. Morgan (Ed.), Handbook of Causal Analysis for Social Research (pp. 245-273). Springer Netherlands. 
Fischer, J. A. V., \& Sousa-Poza, A. (2009). Does job satisfaction improve the health of workers? New evidence using panel data and objective measures of health. Health Economics, 18(1), 71-89.

Friedman, H. L., \& Brown, N. J. L. (2018). Implications of Debunking the "Critical Positivity Ratio" for Humanistic Psychology: Introduction to Special Issue. Journal of Humanistic Psychology, 58(3), 239-261.

Granger, C. W. J. (1969). Investigating Causal Relations by Econometric Models and Cross-spectral Methods. Econometrica: Journal of the Econometric Society, 37(3), 424-438.

Grosz, M. P., Rohrer, J. M., \& Thoemmes, F. (2020). The Taboo Against Explicit Causal Inference in Nonexperimental Psychology. Perspectives on Psychological Science: A Journal of the Association for Psychological Science, 15(5), 1243-1255.

Hamaker, E. L., Kuiper, R. M., \& Grasman, R. P. P. P. (2015). A critique of the cross-lagged panel model. Psychological Methods, 20(1), 102-116.

Hernán, M. A., \& Robins, J. M. (2010). Causal inference: What If. CRC Boca Raton, FL; https://grass.upc.edu/en/seminar/presentation-files/causal-inference/chapters-1-i-2/@@ download/file/BookHernanRobinsCap1_2.pdf

Hernán, M. A., \& Taubman, S. L. (2008). Does obesity shorten life? The importance of well-defined interventions to answer causal questions. International Journal of Obesity , 32 Suppl 3, S8-S14.

Ickovics, J. R., Milan, S., Boland, R., Schoenbaum, E., Schuman, P., Vlahov, D., \& HIV Epidemiology Research Study (HERS) Group. (2006). Psychological resources protect health: 5-year survival and immune function among HIV-infected women from four US cities. AIDS , 20(14), 1851-1860.

Imai, K., Keele, L., \& Yamamoto, T. (2010). Identification, Inference and Sensitivity Analysis for Causal Mediation Effects. Statistical Science: A Review Journal of the Institute of 
Mathematical Statistics, 25(1), 51-71.

Kim, E. S., Park, N., Sun, J. K., Smith, J., \& Peterson, C. (2014). Life satisfaction and frequency of doctor visits. Psychosomatic Medicine, 76(1), 86-93.

Krieger, N., \& Davey Smith, G. (2016). The tale wagged by the DAG: broadening the scope of causal inference and explanation for epidemiology. International Journal of Epidemiology, 45(6), 1787-1808.

Kushlev, K., Heintzelman, S. J., Lutes, L. D., Wirtz, D., Kanippayoor, J. M., Leitner, D., \& Diener, E. (2020). Does Happiness Improve Health? Evidence From a Randomized Controlled Trial. Psychological Science, 31(7), 807-821.

MacKinnon, D. P., \& Pirlott, A. G. (2015). Statistical approaches for enhancing causal interpretation of the $\mathrm{M}$ to $\mathrm{Y}$ relation in mediation analysis. Personality and Social Psychology Review: An Official Journal of the Society for Personality and Social Psychology, Inc, 19(1), 30-43.

Morgan, S. L., \& Winship, C. (2015). Counterfactuals and Causal Inference. Cambridge University Press.

Moskowitz, J. T. (2003). Positive affect predicts lower risk of AIDS mortality. Psychosomatic Medicine, 65(4), 620-626.

Munafò, M. R., \& Davey Smith, G. (2018). Robust research needs many lines of evidence. Nature, 553(7689), 399-401.

Oster, E. (2019). Unobservable Selection and Coefficient Stability: Theory and Evidence. Journal of Business \& Economic Statistics: A Publication of the American Statistical Association, 37(2), 187-204.

Pearl, J. (2018). Does Obesity Shorten Life? Or is it the Soda? On Non-manipulable Causes. Journal of Causal Inference, 6(2), 20182001.

Pearl, J. (2019, October 4). Twitter. https://twitter.com/yudapearl/status/1115877431666253825?s=20 
Pressman, S. D., \& Cohen, S. (2012). Positive emotion word use and longevity in famous deceased psychologists. Health Psychology: Official Journal of the Division of Health Psychology, American Psychological Association, 31(3), 297-305.

Robins, J. M., Rotnitzky, A., \& Scharfstein, D. O. (2000). Sensitivity Analysis for Selection bias and unmeasured Confounding in missing Data and Causal inference models. Statistical Models in Epidemiology, the Environment, and Clinical Trials, 1-94.

Rohrer, J. M. (2018). Thinking Clearly About Correlations and Causation: Graphical Causal Models for Observational Data. Advances in Methods and Practices in Psychological Science, 1(1), 27-42.

Rohrer, J. M., \& Arslan, R. C. (2020). Precise answers to vague questions: Issues with interactions. In PsyArXiv. https://doi.org/10.31234/osf.io/7fm2j

Ryan, O., Bringmann, L. F., \& Schuurman, N. K. (2019). The Challenge of Generating Causal Hypotheses Using Network Models. https://doi.org/10.31234/osf.io/ryg69

Ryff, C. D., Radler, B. T., \& Friedman, E. M. (2015). Persistent psychological well-being predicts improved self-rated health over 9-10 years: Longitudinal evidence from MIDUS. Health Psychology Open, 2(2), 2055102915601582.

Seale, G. S., Berges, I.-M., Ottenbacher, K. J., \& Ostir, G. V. (2010). Change in positive emotion and recovery of functional status following stroke. Rehabilitation Psychology, 55(1), 33-39.

VanderWeele, T. J. (2009). On the distinction between interaction and effect modification. Epidemiology , 20(6), 863-871.

van Geert, P. L. C. (2020). Dynamic Systems, Process and Development. Human Development, 63(3-4), 153-179.

Vanhove, J. (2020). Collinearity isn't a disease that needs curing. https://doi.org/10.31234/osf.io/mv2wx

Voellmin, A., Entringer, S., Moog, N., Wadhwa, P. D., \& Buss, C. (2013). Maternal positive 
affect over the course of pregnancy is associated with the length of gestation and reduced risk of preterm delivery. Journal of Psychosomatic Research, 75(4), 336-340.

Westfall, J., \& Yarkoni, T. (2016). Statistically Controlling for Confounding Constructs Is Harder than You Think. PloS One, 11(3), e0152719.

White, C. A., Uttl, B., \& Holder, M. D. (2019). Meta-analyses of positive psychology interventions: The effects are much smaller than previously reported. PloS One, 14(5), e0216588.

Wootton, R. E., Lawn, R. B., Millard, L. A. C., Davies, N. M., Taylor, A. E., Munafò, M. R., Timpson, N. J., Davis, O. S. P., Davey Smith, G., \& Haworth, C. M. A. (2018). Evaluation of the causal effects between subjective wellbeing and cardiometabolic health: mendelian randomisation study. BMJ , 362, k3788.

Yarkoni, T. (2019). The Generalizability Crisis. In psyarxiv.com > jqw35psyarxiv.com > jqw35. https://doi.org/10.31234/osf.io/jqw35 